\title{
Qualitative Analysis of a Diffusive Ratio-Dependent Holling-Tanner Predator-Prey Model with Smith Growth
}

\author{
Zongmin Yue ${ }^{1}$ and Wenjuan Wang ${ }^{2}$ \\ ${ }^{1}$ Faculty of Science, Shaanxi University of Science and Technology, Xian 710021, China \\ ${ }^{2}$ Department of Biostatistics and Computational Biology, University of Rochester Medical Center, 601 Elmwood Avenue, \\ P.O. Box 630, Rochester, NY 14642, USA
}

Correspondence should be addressed to Zongmin Yue; joanna_yzm@163.com

Received 28 January 2013; Accepted 15 February 2013

Academic Editor: Yonghui Xia

Copyright (c) 2013 Z. Yue and W. Wang. This is an open access article distributed under the Creative Commons Attribution License, which permits unrestricted use, distribution, and reproduction in any medium, provided the original work is properly cited.

\begin{abstract}
We investigated the dynamics of a diffusive ratio-dependent Holling-Tanner predator-prey model with Smith growth subject to zero-flux boundary condition. Some qualitative properties, including the dissipation, persistence, and local and global stability of positive constant solution, are discussed. Moreover, we give the refined a priori estimates of positive solutions and derive some results for the existence and nonexistence of nonconstant positive steady state.
\end{abstract}

\section{Introduction}

In order to precisely describe the real ecological interactions between species such as mite and spider mite, lynx and hare, sparrow and sparrow hawk, and some other species [1,2], Robert May developed a prey-predator model of Holling-type functional response $[3,4]$ to describe the predation rate and Leslie's formulation $[5,6]$ to describe predator dynamics. This model is known as Holling-Tanner model for prey-predator interaction, which takes the form of

$$
\begin{gathered}
\frac{d N}{d t}=r N\left(1-\frac{N}{K}\right)-\frac{m N P}{a+N}, \\
\frac{d P}{d t}=s P\left(1-\frac{h P}{N}\right),
\end{gathered}
$$

where $N(t)$ and $P(t)$ stand for prey and predator population (density) at any instant of time $t . r, K, m, a, s, h$ are positive constants that stand for prey intrinsic growth rate, carrying capacity, capturing rate, half capturing saturation constant, predator intrinsic growth rate, and conversion rate of prey into predators biomass, respectively.

The dynamics of model (1) has been considered in many articles. For example, Hsu and Huang [7] obtained some results on the global stability of the positive equilibrium, more precisely, under the conditions which local stability of the positive equilibrium implies its global stability. Gasull and coworkers [8] investigated the conditions of the asymptotic stability of the positive equilibrium which does not imply global stability. Sáez and González-Olivares [9] showed the asymptotic stability of a positive equilibrium and gave a qualitative description of the bifurcation curve.

Recently, there is a growing explicit biological and physiological evidence [10-12] that in many situations, especially, when the predator has to search for food (and therefore has to share or compete for food), a more suitable general predator-prey theory should be based on the so-called radiodependent theory which can be roughly stated as that the per capital predator growth rate should be a function of the ratio of prey to predator abundance, and so would be the socalled predator functional responses [13]. This is supported by numerous fields and laboratory experiments and observations $[14,15]$. Generally, a ratio-dependent Holling-Tanner predator-prey model takes the form of

$$
\begin{gathered}
\frac{d N}{d t}=r N\left(1-\frac{N}{K}\right)-\frac{m N P}{N+a P}, \\
\frac{d P}{d t}=s P\left(1-\frac{h P}{N}\right) .
\end{gathered}
$$

For model (2), in [13], the authors investigated the effect of time delays on the stability of the model and discussed 
the local asymptotic stability and the Hopf-bifurcation. Liang and Pan [16] have studied the local and global asymptotic stability of the coexisting equilibrium point and obtained the conditions for the Poincaré-Andronov-Hopf-bifurcating periodic solution. M. Banerjee and S. Banerjee [17] have studied the local asymptotic stability of the equilibrium point and obtained the conditions for the occurrence of the TuringHopf instability for PDE model. It is shown that prey and predator populations exhibit spatiotemporal chaos resulting from temporal oscillation of both the population and spatial instability.

On the other hand, an implicit assumption contained in the logistic equation

$$
\frac{d N}{d t}=r N\left(1-\frac{N}{K}\right)
$$

is that the average growth rate $N^{\prime}(t) / N$ is a linear function of the density $N(t)$. It has been shown that this assumption is not realistic for a food-limited population under the effects of environmental toxicants. The following alternative model has been proposed by several authors [18-23] for the dynamics of a population where the growth limitations are based upon the proportion of available resources not utilized:

$$
\frac{d N}{d t}=r N \frac{K-N}{K+c N},
$$

where $r / c$ is the replacement of mass in the population at $K$. Equation (4) takes into account both environmental and food chain effects of toxicant stress.

Based on the above discussions, in this paper, we rigorously consider the radio-dependent Holling-Tanner model with Smith growth that takes the form of

$$
\begin{gathered}
\frac{d N}{d t}=r N \frac{K-N}{K+c N}-\frac{m N P}{a P+N}, \\
\frac{d P}{d t}=s P\left(1-\frac{h P}{N}\right) .
\end{gathered}
$$

Also considering the spatial dispersal and environmental heterogeneity, in this paper, we study the following generalized reaction-diffusion system for model (5):

$$
\begin{gathered}
\frac{\partial N}{d t}=r N \frac{K-N}{K+c N}-\frac{m N P}{a P+N}+d_{1} \Delta N, \quad x \in \Omega, t>0, \\
\frac{\partial P}{d t}=s P\left(1-\frac{h P}{N}\right)+d_{2} \Delta P, \quad x \in \Omega, t>0, \\
\frac{\partial N}{\partial \nu}=\frac{\partial P}{\partial \nu}=0, \quad x \in \partial \Omega, t>0,
\end{gathered}
$$

where $\Omega \subset \mathbb{R}^{n}(n \geq 1)$ is a bounded domain with a smooth boundary $\partial \Omega$ and $\nu$ is the outward unit normal vector on $\partial \Omega$. The nonnegative constants $d_{1}$ and $d_{2}$ are the diffusion coefficients of $N$ and $P$, respectively. The zero-flux boundary condition indicates that predator-prey system is self-contained with zero population flux across the boundary. From the standpoint of biology, we are interested only in the dynamics of model (6) in the closed first quadrant
$\mathbb{R}_{+}^{2}=\{(N, P): N \geq 0, P \geq 0\}$. Thus, we consider only the biologically meaningful initial conditions

$$
N(x, 0)=N_{0}(x)>0, \quad P(x, 0)=P_{0}(x)>0, \quad x \in \Omega,
$$

which are continuous functions due to its biological sense. Straightforward computation shows that model (6) are continuous and Lipschizian in $\mathbb{R}_{+}^{2}$ if we redefine that when

$$
\frac{\partial N}{\partial t}=\frac{\partial P}{\partial t}=0, \quad \text { if }(N, P)=(0,0)
$$

Hence, the solution of model (6) with positive initial conditions exists and is unique.

The stationary problem of model (6), which may display the dynamical behavior of solutions to model (6) as time goes to infinity, satisfies the following elliptic system:

$$
\begin{gathered}
-d_{1} \Delta N=r N \frac{K-N}{K+c N}-\frac{m N P}{N+a P}, \quad x \in \Omega, t>0, \\
-d_{2} \Delta P=s P\left(1-\frac{h P}{N}\right), \quad x \in \Omega, t>0, \\
\frac{\partial N}{\partial \nu}=\frac{\partial P}{\partial \nu}=0, \quad x \in \partial \Omega, t>0, \\
N(x, 0)=N_{0}(x)>0, \quad P(x, 0)=P_{0}(x)>0, \quad x \in \Omega .
\end{gathered}
$$

Simple computation shows that if $m<r(a+h)$, then model (6) and (9) possess a unique positive constant solution, denoted by $E^{*}=\left(N^{*}, P^{*}\right)$, where

$$
N^{*}=\frac{K(a r+h r-m)}{a r+c m+h r}, \quad P^{*}=\frac{1}{h} N^{*}
$$

In addition, $(K, 0)$ is the second nonnegative constant steady state of model (6) and (9).

The rest of the paper is organized as follows. In Section 2, we investigate the lager time behavior of model (6), including the dissipation, persistence property, and local and global stability of positive constant solution $E^{*}$. In Section 3, we first give a priori upper and lower bounds for positive solutions of model (9), and then we deal with existence and nonexistence of nonconstant positive solutions of model (9), which imply some certain conditions under which the pattern happens or not.

\section{Large Time Behavior of Solution to Model (6)}

In this section, the dissipation and persistence properties are studied for solution of model (6). Moreover, the local and global asymptotic stability of positive constant solution $E^{*}=$ $\left(N^{*}, P^{*}\right)$ are investigated. 


\subsection{The Properties of Dissipation and}

Persistence of Solution to Model (6)

Theorem 1. All the solutions of model (6) are nonnegative and defined for all $t>0$. Furthermore, the nonnegative solution $(N, P)$ of model $(6)$ satisfies

$$
\lim _{t \rightarrow \infty} \sup _{\bar{\Omega}} \max _{\bar{\Omega}} N(\cdot, t) \leq K, \quad \lim _{t \rightarrow \infty} \sup _{\bar{\Omega}} \max _{\bar{\Omega}} P(\cdot, t) \leq \frac{K}{h} .
$$

Proof. The nonnegativity of the solution of model (6) is clear since the initial value is nonnegative. We only consider the latter of the theorem.

Note that $N$ satisfies

$$
\begin{gathered}
\frac{\partial N}{\partial t}-d_{1} \Delta N \leq \frac{r N(K-N)}{K+c N}, \quad x \in \Omega, t>0, \\
\frac{\partial N}{\partial \nu}=0, \quad x \in \partial \Omega, t>0, \\
N(x, 0)=N_{0}(x), \quad x \in \Omega .
\end{gathered}
$$

Let $z(t)$ be a solution of the ordinary differential equation:

$$
\begin{gathered}
\dot{z}(t)=\frac{r N(K-N)}{K+c N}, \quad x \in \Omega, t>0, \\
z(0)=\max _{\bar{\Omega}} N(x, 0)>0 .
\end{gathered}
$$

Then, $\lim _{t \rightarrow \infty} z(t)=K$. From the comparison principle, one can get $N(x, t) \leq z(t)$; hence,

$$
\lim _{t \rightarrow \infty} \sup _{\bar{\Omega}} \max _{\bar{\Omega}} N(x, t) \leq K .
$$

As a result, for any $\varepsilon>0$, there exists $t_{0}>0$, such that $N(x, t) \leq K+\varepsilon$ for all $x \in \bar{\Omega}$ and $t \geq t_{0}$. Hence, $P(x, t)$ is a lower solution

$$
\begin{gathered}
\frac{\partial z}{\partial t}-d_{2} \Delta z=s z\left(1-\frac{h w}{K+\varepsilon}\right), \quad x \in \Omega, t>t_{0} \\
\frac{\partial z}{\partial v}=0, \quad x \in \partial \Omega, t>t_{0}, \\
z\left(x, t_{0}\right)=P\left(x, t_{0}\right) .
\end{gathered}
$$

Let $P(t)$ be the unique positive solution of problem

$$
\begin{gathered}
\dot{w}(t)=s w\left(1-\frac{h w}{K+\varepsilon}\right), \quad t>t_{0}, \\
w\left(t_{0}\right)=\max _{\bar{\Omega}} P\left(x, t_{0}\right) .
\end{gathered}
$$

Then, $P(t)$ is an upper solution of (15). As $\lim _{t \rightarrow \infty} P(t)=(K+$ $\varepsilon) / h$, we get from the comparison principle that

$$
\limsup _{t \rightarrow \infty} \max _{\bar{\Omega}} P(x, t) \leq \frac{K+\varepsilon}{h},
$$

which implies the second assertion by the arbitrariness of $\varepsilon>$ 0 . This ends the proof.
Definition 2 (see [24]). The spatial model (6) is said to have the persistence property if for any nonnegative initial data $\left(N_{0}(x), P_{0}(x)\right)$, there exists a positive constant $\varepsilon=\varepsilon\left(N_{0}, P_{0}\right)$, such that the corresponding solution $(N, P)$ of model (6) satisfies

$$
\liminf _{t \rightarrow \infty} \min _{\bar{\Omega}} N(x, t) \geq \varepsilon, \quad \liminf _{t \rightarrow \infty} \min _{\bar{\Omega}} P(x, t) \geq \varepsilon .
$$

Theorem 3. If $m(1+c)<$ ar, then model (6) has the persistence property.

Proof. Let $N(x, t)$ be an upper solution of the following problem:

$$
\begin{gathered}
\frac{\partial z}{\partial t}-d_{1} \Delta z=z\left(\frac{r}{1+c}-\frac{m}{a}-\frac{r z}{K(1+c)}\right), \quad x \in \Omega, t>T, \\
\frac{\partial z}{\partial \nu}=0, \quad x \in \partial \Omega, t>T, \\
z(x, T)=N_{0}(x, T) \geq 0, \quad x \in \bar{\Omega} .
\end{gathered}
$$

Let $N(t)$ be the unique positive solution to the following problem:

$$
\begin{gathered}
\frac{d w}{d t}=w\left(\frac{r}{1+c}-\frac{m}{a}-\frac{r w}{K(1+c)}\right), \quad t>T, \\
w(T)=\max _{\bar{\Omega}} N_{0}(x, T) \geq 0 .
\end{gathered}
$$

Due to $m(1+c)<$ ar, we have that $\lim _{t \rightarrow \infty} w(t)=K($ ar $m(1+c)) / a r$. By comparison, it follows that

$$
\liminf _{t \rightarrow \infty} \min _{\bar{\Omega}} N(x, t) \geq \frac{K(a r-m(1+c))}{a r} \triangleq \eta .
$$

Hence, $N(x, t)>\eta-\varepsilon$ for $t>T$ and $x \in \bar{\Omega}$.

Similarly, by the second equation of model (6), we have that $P(x, t)$ is an upper solution of problem

$$
\begin{gathered}
\frac{\partial z}{\partial t}-d_{2} \Delta z=s z\left(1-\frac{h z}{\eta-\varepsilon}\right), \quad x \in \Omega, t>T, \\
\frac{\partial z}{\partial \nu}=0, \quad x \in \partial \Omega, t>T, \\
z(x, T)=P_{0}(x, T) \geq 0, \quad x \in \bar{\Omega} .
\end{gathered}
$$

Let $P(t)$ be the unique positive solution to the following problem:

$$
\begin{gathered}
\frac{d w}{d t}=s z\left(1-\frac{h z}{\eta-\varepsilon}\right), \quad t>T, \\
w(T)=\max _{\bar{\Omega}} P_{0}(x, T) \geq 0 .
\end{gathered}
$$

Then, $\lim _{t \rightarrow \infty} w(t)=\eta / h$ for the arbitrariness of $\varepsilon$, and an application of the comparison principle gives

$$
\liminf _{t \rightarrow \infty} \min _{\bar{\Omega}} P(x, t) \geq \frac{\eta}{h} .
$$

The proof is complete. 
2.2. The Local Stability of the Constant Steady State. In this subsection, we shall analyze the asymptotical stability of the positive constant solution $E^{*}$ for model (6). Before developing our argument, let us set up the following notations.

(i) Let $0=\mu_{0}<\mu_{1}<\mu_{2}<\cdots \rightarrow \infty$ be the eigenvalues of the operator $-\Delta$ on $\Omega$ with the zero-flux boundary condition;

(ii) Let $E(\mu)=\left\{\phi \mid-\Delta \phi=\mu \phi\right.$ in $\Omega, \partial_{\nu} \phi=0$ on $\left.\partial \Omega\right\}$ with $\mu \in \mathbb{R}^{1}$;

(iii) Let $\left\{\phi_{i j} \mid j=1, \ldots, \operatorname{dim} E\left(\mu_{i}\right)\right\}$ be an orthonormal basis of $E\left(\mu_{i}\right)$, and $\mathbf{X}_{i j}=\left\{\mathbf{c} \phi_{i j} \mid \mathbf{c} \in \mathbb{R}^{2}\right\}$;

(iv) Let

$$
\mathbf{X}=\left\{(N, P) \in\left[H^{2}(\Omega)\right]^{2} \mid \partial_{\nu} N=\partial_{\nu} P=0 \text { on } \partial \Omega\right\},
$$

then

$$
\mathbf{X}=\bigoplus_{i=1}^{\infty} \mathbf{x}_{i}
$$

where $\mathbf{X}_{i}=\bigoplus_{j=1}^{\operatorname{dim} E\left(\mu_{i}\right)} \mathbf{X}_{i j}$.

Theorem 4. Assume that

$$
m(a r+c m+2 h r-a c r)<r(a+h)^{2}(r+s+c s)
$$

and the first eigenvalues $\mu_{1}$ of the Dirichlet operator subject to zero-flux boundary conditions satisfy

$$
\mu_{1}>\max \left\{\frac{m(a r+c m+2 h r-a c r)-r^{2}(a+h)^{2}}{d_{1} r(1+c)(a+h)^{2}}-\frac{s}{d_{2}}, 0\right\} .
$$

Then the positive constant solution $E^{*}$ of model (6) is locally asymptotically stable.

Proof. Define $\mathscr{L}: \mathbf{X} \rightarrow C(\bar{\Omega}) \times C(\bar{\Omega})$ by

$$
\mathscr{L}=\left(\begin{array}{cc}
d_{1} \Delta+J_{1} & -\frac{m h^{2}}{(a+h)^{2}} \\
-\frac{s}{h} & d_{2} \Delta-s
\end{array}\right),
$$

where $J_{1}=\left(m(a r+c m+2 h r-a c r)-r^{2}(a+h)^{2}\right) / r(1+c)(a+h)^{2}$.

For each $i=0,1,2, \ldots, \mathbf{X}_{i}$ is invariant under the operator $\mathscr{L}$, and $\lambda$ is an eigenvalue of this operator on $\mathbf{X}_{i}$ if and only if it is an eigenvalue of the following matrix:

$$
A_{i}=\left(\begin{array}{cc}
-d_{1} \mu_{i}+J_{1} & -\frac{m h^{2}}{(a+h)^{2}} \\
-\frac{s}{h} & -d_{2} \mu_{i}-s
\end{array}\right) .
$$

Moreover,

$$
\operatorname{det}\left(\lambda I-A_{i}\right)=\lambda^{2}-\operatorname{tr}\left(A_{i}\right) \lambda+\operatorname{det}\left(A_{i}\right),
$$

where

$$
\begin{gathered}
\operatorname{det}\left(A_{i}\right)=d_{1} d_{2} \mu_{i}^{2}+\left(d_{1} s-d_{2} J_{1}\right) \mu_{i} \\
+\frac{s(a r+h r-m)(a r+h r+c m)}{r(1+c)(a+h)^{2}}, \\
\operatorname{tr}\left(A_{i}\right)=-\left(d_{1}+d_{2}\right) \mu_{i}+J_{1}-s .
\end{gathered}
$$

In view of (27) and (28), we have $\operatorname{det}\left(A_{i}\right)>0>\operatorname{tr}\left(A_{i}\right)$ for any $i \geq 0$. Therefore, the eigenvalues of the matrix $A_{i}$ have negative real parts.

In the following, we prove that there exists $\delta>0$ such that

$$
\operatorname{Re}\left\{\lambda_{i 1}\right\} \leq-\delta, \quad \operatorname{Re}\left\{\lambda_{i 2}\right\} \leq-\delta
$$

Let $\lambda=\mu_{i} \xi$, then

$$
\widetilde{\varphi}_{i}(\lambda) \triangleq \mu_{i}^{2} \xi^{2}-\operatorname{tr}\left(A_{i}\right) \mu_{i} \xi+\operatorname{det}\left(A_{i}\right) .
$$

Since $\mu_{i} \rightarrow \infty$ as $i \rightarrow \infty$, it follows that

$$
\lim _{i \rightarrow \infty} \frac{\widetilde{\varphi}_{i}(\lambda)}{\mu_{i}^{2}}=\xi^{2}+\left(d_{1}+d_{2}\right) \xi+d_{1} d_{2}
$$

By the Routh-Hurwitz criterion, it follows that the two roots $\xi_{1}, \xi_{2}$ of $\tilde{\varphi}_{i}(\lambda)=0$ all have negative real parts. Thus, let $\widetilde{d}=\min \left\{d_{1}, d_{2}\right\}$, we have that $\operatorname{Re}\left\{\xi_{1}\right\}, \operatorname{Re}\left\{\xi_{2}\right\} \leq-\widetilde{d}$. By continuity, we see that there exists $i_{0}$ such that the two roots $\xi_{i 1}, \xi_{i 2}$ of $\widetilde{\varphi}_{i}(\lambda)=0$ satisfy $\operatorname{Re}\left\{\xi_{i 1}\right\} \leq-\tilde{d} / 2, \operatorname{Re}\left\{\xi_{i 2}\right\} \leq-\widetilde{d} / 2$, for all $i \geq i_{0}$. In turn, $\operatorname{Re}\left\{\lambda_{i 1}\right\}, \operatorname{Re}\left\{\lambda_{i 2}\right\} \leq-\mu_{i} \tilde{d} / 2 \leq-\tilde{d} / 2$, for all $i \geq i_{0}$.

Let $-\widetilde{\delta}=\max _{1 \leq i \leq i_{0}}\left\{\operatorname{Re}\left\{\lambda_{i 1}\right\}, \operatorname{Re}\left\{\lambda_{i 2}\right\}\right\}$, then $\widetilde{\delta}>0$ and (33) hold for $\delta=\min \{\widetilde{\delta}, \widetilde{d} / 2\}$. Consequently, the spectrum of $\mathscr{L}$ which consists of eigenvalues, lies in $\{\operatorname{Re} \lambda \leq-\delta\}$. In the sense of [25], we obtain that the positive constant solution $E^{*}=$ $\left(N^{*}, P^{*}\right)$ of model (6) is uniformly asymptotically stable. This ends the proof.

2.3. The Global Stability of the Constant Solution. This subsection is devoted to the global stability of the constant solution $E^{*}$ for model (6).

Theorem 5. Assume that the following hold:

$$
\begin{aligned}
& \text { (A1) } m(1+c)<a r ; \\
&\text { (A2) }) h\left(K+c N^{*}\right)\left(N^{*}+2 P^{*}\right)+\left(K+c N^{*}\right)\left(N^{*}+a P^{*}\right)(a+h) \leq \\
& 2 \eta r\left(N^{*}+a P^{*}\right)(a+h) ; \\
& \text { (A3) } h K N^{*} \leq \eta(2 h-1)\left(N^{*}+a P^{*}\right)(a+h),
\end{aligned}
$$

where $\eta=K($ ar $-m(1+c)) /$ ar. Then the constant solution $E^{*}$ is globally asymptotically stable.

Proof. In order to give the proof, we need to construct a Lyapunov function. Define

$$
\begin{aligned}
V(N, P) & =\int_{N^{*}}^{N} \frac{\xi-N^{*}}{\xi} d \xi+\int_{P^{*}}^{P} \frac{\zeta-P^{*}}{\zeta} d \zeta, \\
E(t) & =\int_{\Omega} V(N(x, t), P(x, t)) d x .
\end{aligned}
$$


We note that $E(t)$ is nonnegative, $E(t)=0$ if and only if $(N(x, t), P(x, t))=\left(N^{*}, P^{*}\right)$. Furthermore, by simple computations, it follows that

$$
\begin{aligned}
\frac{d E(t)}{d t}= & \int_{\Omega}\left(V_{N}(N(x, t), P(x, t)) N_{t}\right. \\
& \left.+V_{P}(N(x, t), P(x, t)) P_{t}\right) d x \\
= & \int_{\Omega}\left(\frac{d_{1}\left(N-N^{*}\right)}{N} \Delta N+\frac{d_{2}\left(P-P^{*}\right)}{P} \Delta P\right) d x \\
& +\int_{\Omega}\left(\left(N-N^{*}\right)\left(\frac{r(K-N)}{K+c N}-\frac{m P}{N+a P}\right)\right. \\
= & -\int_{\Omega}\left(\frac{d_{1} N^{*}}{N^{2}}|\nabla N|^{2}+\frac{d_{2} P^{*}}{P^{2}}|\nabla P|^{2}\right) d x+I(t),
\end{aligned}
$$

where

$$
\begin{array}{r}
I(t)=\int_{\Omega}\left(( N - N ^ { * } ) ^ { 2 } \left(-\frac{r K(1+c)}{\left(K+c N^{*}\right)(K+c N)}\right.\right. \\
\left.\left.+\frac{P^{*}}{\left(N^{*}+a P^{*}\right)(N+a P)}\right)\right) d x \\
+\int_{\Omega}\left(\left(N-N^{*}\right)\left(P-P^{*}\right)\right. \\
\quad \times\left(\frac{1}{N}-\frac{N^{*}}{\left(N^{*}+a P^{*}\right)(N+a P)}\right) \\
\left.-\frac{h}{N}\left(P-P^{*}\right)^{2}\right) d x .
\end{array}
$$

Set $\varphi=N-N^{*}, \phi=P-P^{*}$. We have

$$
\begin{aligned}
I(t)=- & \int_{\Omega}\left(\frac{r K(1+c)}{\left(K+c N^{*}\right)(K+c N)}\right. \\
& \left.\quad-\frac{N^{*}+2 P^{*}}{2\left(N^{*}+a P^{*}\right)(N+a P)}-\frac{1}{2 N}\right) \varphi^{2} d x \\
- & \int_{\Omega}\left(\frac{2 h-1}{2 N}-\frac{N^{*}}{2\left(N^{*}+a P^{*}\right)(N+a P)}\right) \phi^{2} d x \\
- & \int_{\Omega}\left(\frac{1}{2 N}(\varphi-\phi)^{2}+\frac{N^{*}}{2\left(N^{*}+a P^{*}\right)(N+a P)}\right. \\
& \left.\times(\varphi+\phi)^{2}\right) d x
\end{aligned}
$$

$$
\begin{aligned}
\leq- & \int_{\Omega}\left(\frac{r K(1+c)}{\left(K+c N^{*}\right)(K+c N)}\right. \\
& \left.\quad-\frac{N^{*}+2 P^{*}}{2\left(N^{*}+a P^{*}\right)(N+a P)}-\frac{1}{2 N}\right) \varphi^{2} d x \\
- & \int_{\Omega}\left(\frac{2 h-1}{2 N}-\frac{N^{*}}{2\left(N^{*}+a P^{*}\right)(N+a P)}\right) \phi^{2} d x .
\end{aligned}
$$

By virtue of Theorems 1 and 3 and under the assumption of Theorem, we have

$$
\begin{aligned}
& \frac{r K(1+c)}{\left(K+c N^{*}\right)(K+c N)}-\frac{N^{*}+2 P^{*}}{2\left(N^{*}+a P^{*}\right)(N+a P)}-\frac{1}{2 N} \\
& \geq \frac{r}{K+c N^{*}}-\frac{h\left(N^{*}+2 P^{*}\right)}{2 \eta\left(N^{*}+a P^{*}\right)(a+h)}-\frac{1}{2 \eta} \\
& =\left(2 \eta r\left(N^{*}+a P^{*}\right)(a+h)\right. \\
& -h\left(K+c N^{*}\right)\left(N^{*}+2 P^{*}\right) \\
& \left.-\left(K+c N^{*}\right)\left(N^{*}+a P^{*}\right)(a+h)\right) \\
& \times\left(2 \eta\left(K+c N^{*}\right)\left(N^{*}+a P^{*}\right)(a+h)\right)^{-1} \\
& \geq 0 \text {, } \\
& \frac{2 h-1}{2 N}-\frac{N^{*}}{2\left(N^{*}+a P^{*}\right)(N+a P)} \\
& \geq \frac{2 h-1}{2 K}-\frac{h N^{*}}{2 \eta\left(N^{*}+a P^{*}\right)(a+h)} \\
& =\frac{\eta(2 h-1)\left(N^{*}+a P^{*}\right)(a+h)-h K N^{*}}{2 K \eta\left(N^{*}+a P^{*}\right)(a+h)} \\
& \geq 0 \text {. }
\end{aligned}
$$

As a result, we have $I(t) \leq 0$. Thus $d E(t) / d t \leq 0$, which implies the desired assertion. The proof is completed.

\section{A Priori Estimates and Existence of Nonconstant Positive Solution}

In this section, we will deduce a priori estimates of positive upper and lower bounds for positive solution of model (9). Then, based on a priori estimates, we discuss the existence of nonconstant positive solution of model (9) for certain parameter ranges.

3.1. A Priori Estimates. In order to obtain the desired bound, we recall the following two lemmas which are due to Lin et al. [26] and Lou and Ni [27], respectively. 
Lemma 6 (Harnack's inequality [26]). Assume that $c \in C(\bar{\Omega})$ and let $w \in C^{2}(\Omega) \cap C^{1}(\bar{\Omega})$ be a positive solution to

$$
\Delta w(x)+c(x) w(x)=0 \quad \text { in } \Omega, \quad \frac{\partial w}{\partial \nu}=0 \quad \text { on } \partial \Omega .
$$

Then there exists a positive constant $C^{*}=C^{*}\left(\|c\|_{\infty}\right)$ such that

$$
\max _{\bar{\Omega}} w \leq C^{*} \min _{\bar{\Omega}} w .
$$

Lemma 7 (maximum principle [27]). Let $\Omega$ be a bounded Lipschitz domain in $\mathbb{R}^{2}$ and $g \in C(\bar{\Omega} \times \mathbb{R})$.

(a) Assume that $w \in C^{2}(\Omega) \cap C^{1}(\bar{\Omega})$ and satisfies

$$
\Delta w(x)+g(x, w(x)) \geq 0 \quad \text { in } \Omega, \quad \frac{\partial w}{\partial \nu} \leq 0 \quad \text { on } \partial \Omega .
$$

$$
\text { If } w\left(x_{0}\right)=\max _{\bar{\Omega}} w(x) \text {, then } g\left(x_{0}, w\left(x_{0}\right)\right) \geq 0 \text {. }
$$

(b) Assume that $w \in C^{2}(\Omega) \cap C^{1}(\bar{\Omega})$ and satisfies

$$
\Delta w(x)+g(x, w(x)) \leq 0 \quad \text { in } \Omega, \quad \frac{\partial w}{\partial \nu} \geq 0 \quad \text { on } \partial \Omega
$$

$$
\text { If } w\left(x_{0}\right)=\min _{\bar{\Omega}} w(x), \text { then } g\left(x_{0}, w\left(x_{0}\right)\right) \leq 0 .
$$

For convenience, let us denote the constants $a, c, h, m, K, r, s$ collectively by $\Lambda$. The positive constants $C, C, \bar{C}$, and so forth will depend only on the domain $\Omega$ and $\Lambda$. Now, we can state the main result which will play a critical role in Section 3.3.

Theorem 8. For any positive solution $(N, P)$ of model (9),

$$
\max _{\bar{\Omega}} N(x) \leq K, \quad \max _{\bar{\Omega}} P(x) \leq \frac{K}{h} .
$$

Proof. Assume that $(N, P)$ is a positive solution of model (9). Set

$$
N\left(x_{1}\right)=\max _{\bar{\Omega}} N(x) .
$$

Then, by Lemma 7, it follows from the first equation of (9) that

$$
\frac{r N\left(x_{1}\right)\left(K-N\left(x_{1}\right)\right)}{K+c N\left(x_{1}\right)} \geq \frac{m N\left(x_{1}\right) P\left(x_{1}\right)}{N\left(x_{1}\right)+a P\left(x_{1}\right)}>0 .
$$

This clearly gives $N\left(x_{1}\right)<K$. $\bar{\Omega}$.

Since $0<P(x) \leq(1 / h)\|N(x)\|_{\infty}$, we have $P(x) \leq K / h$ in

Theorem 9. Let $d$ be a fix positive constant. Then there exists positive constant $\underline{C}=\underline{C}(\Lambda, d)$ such that if $d_{1}, d_{2}>d$, any positive solution $(\bar{N}, P)$ of model (6) satisfies

$$
\min _{\bar{\Omega}} N(x) \geq \underline{C}, \quad \min _{\bar{\Omega}} P(x) \geq \underline{C} .
$$

Proof. Let

$$
\begin{aligned}
& N\left(x_{0}\right)=\min _{\bar{\Omega}} N(x), \\
& P\left(y_{0}\right)=\min _{\bar{\Omega}} P(x), \\
& P\left(y_{1}\right)=\max _{\bar{\Omega}} P(x) .
\end{aligned}
$$

By Lemma 7, it is clear that

$$
\begin{gathered}
\frac{r\left(K-N\left(x_{0}\right)\right)}{K+c N\left(x_{0}\right)}-\frac{m P\left(x_{0}\right)}{N\left(x_{0}\right)+a P\left(x_{0}\right)} \leq 0, \\
1-\frac{h P\left(y_{0}\right)}{N\left(y_{0}\right)} \leq 0, \\
1-\frac{h P\left(y_{1}\right)}{N\left(y_{1}\right)} \geq 0 .
\end{gathered}
$$

So, we have

$$
\begin{gathered}
\frac{1}{h} N\left(x_{0}\right) \leq \frac{1}{h} N\left(y_{0}\right) \leq P\left(y_{0}\right), \\
P\left(y_{1}\right) \leq \frac{1}{h} N\left(y_{1}\right) \leq \frac{1}{h} \max _{\bar{\Omega}} N(x) .
\end{gathered}
$$

Since $m\left(K+c N\left(x_{0}\right)\right) /\left(N\left(x_{0}\right)+a P\left(x_{0}\right)\right) \leq C$ with $C>0$, then, by virtue of (52), we derive

$$
\begin{aligned}
K-N\left(x_{0}\right) & \leq \frac{m P\left(x_{0}\right)\left(K+c N\left(x_{0}\right)\right)}{r\left(N\left(x_{0}\right)+a P\left(x_{0}\right)\right)} \leq \frac{C}{r} P\left(x_{0}\right) \leq \frac{C}{r} P\left(y_{1}\right) \\
& \leq \frac{C}{h r} \max _{\bar{\Omega}} N(x),
\end{aligned}
$$

which implies that

$$
K \leq \min _{\bar{\Omega}} N(x)+\frac{C}{h r} \max _{\bar{\Omega}} N(x) .
$$

Define $c(x)=d_{1}^{-1}(r(K-N) /(K+c N)-m P /(N+a P))$, then $N$ satisfies

$$
\Delta N(x)+c(x) N(x)=0 \quad \text { in } \Omega, \quad \frac{\partial N}{\partial \nu}=0 \quad \text { on } \partial \Omega .
$$

Therefore, we have

$$
\max _{\bar{\Omega}} N(x) \leq C^{*} \min _{\bar{\Omega}} N(x),
$$

herein a positive constant $C^{*}=C^{*}\left(\|c\|_{\infty}\right)$. Hence, we obtain

$$
\min _{\bar{\Omega}} N(x) \geq \frac{h K r}{h r+C C^{*}} .
$$

It follows from (51) that

$$
\min _{\bar{\Omega}} P(x) \geq \frac{K r}{h r+C C^{*}} .
$$

The proof is completed. 
3.2. Nonexistence of the Nonconstant Positive Solutions. Note that $\mu_{1}$ is the smallest positive eigenvalues of the operator $-\Delta$ in $\Omega$ subject to the zero-flux boundary condition. Now, using the energy estimates, we can claim the following results.

Theorem 10. Let $D>s / \mu_{1}$ be a fixed positive constant. Then there exists a positive constant $d^{*}=d^{*}(\Lambda, D)$ such that model (9) has no positive nonconstant solution provided that $d_{1}>d^{*}$ and $d_{2}>D$.

Proof. Let $(N, P)$ be any positive solution of model (9) and denote $\bar{g}=|\Omega|^{-1} \int_{\Omega} g d x$. Then

$$
\int_{\Omega}(N-\bar{N}) d x=\int_{\Omega}(P-\bar{P}) d x=0
$$

Then, multiplying the first equation of model (9) by $(N-\bar{N})$, integrating over $\Omega$, we have that

$$
\begin{aligned}
d_{1} \int_{\Omega}|\nabla(N-\bar{N})|^{2} d x \\
=\int_{\Omega} \frac{r N(K-N)(N-\bar{N})}{K+c N} d x \\
\quad-\int_{\Omega} \frac{m N P(N-\bar{N})}{N+a P} d x \\
\leq \int_{\Omega} r N\left(1-\frac{N}{K}\right)(N-\bar{N}) d x \\
\quad-\int_{\Omega} \frac{a m \bar{P} P(N-\bar{N})^{2}}{(N+a P)(\bar{N}+a \bar{P})} d x \\
\quad-\int_{\Omega} \frac{m \bar{N} N(N-\bar{N})(P-\bar{P})}{(N+a P)(\bar{N}+a \bar{P})} d x \\
\leq \int_{\Omega}\left(r(N-\bar{N})^{2}+m|N-\bar{N}||P-\bar{P}|\right) d x .
\end{aligned}
$$

In a similar manner, we multiply the second equation in model (9) by $(P-\bar{P})$ to have

$$
\begin{aligned}
d_{2} \int_{\Omega} & |\nabla(P-\bar{P})|^{2} d x \\
= & \int_{\Omega} s P\left(1-\frac{h P}{N}\right)(P-\bar{P}) d x \\
& =\int_{\Omega} s(P-\bar{P})
\end{aligned}
$$

$$
\begin{gathered}
\times\left(P-\bar{P}-\frac{h(P+\bar{P})(P-\bar{P})}{N}\right. \\
\left.+\frac{h \bar{P}^{2}(N-\bar{N})}{\bar{N} N}\right) d x \\
\leq \int_{\Omega} s\left((P-\bar{P})^{2}+\frac{h \bar{P}^{2}}{\bar{N} N}|N-\bar{N}||P-\bar{P}|\right) d x .
\end{gathered}
$$

By the $\varepsilon$-Young inequality and the Poincare inequality, we obtain that

$$
\begin{gathered}
\int_{\Omega}\left(d_{1}|\nabla(N-\bar{N})|^{2}+d_{2}|\nabla(P-\bar{P})|^{2}\right) d x \\
\leq \int_{\Omega}\left(r(N-\bar{N})^{2}+2 M(N-\bar{N})(P-\bar{P})\right. \\
\left.\quad+s(P-\bar{P})^{2}\right) d x \\
\leq \frac{1}{\mu_{1}} \int_{\Omega}\left(|\nabla(N-\bar{N})|^{2}\left(r+\frac{M}{\epsilon}\right)\right. \\
\left.+|\nabla(P-\bar{P})|^{2}(s+\epsilon M)\right) d x
\end{gathered}
$$

for some positive constant $M$ and an arbitrary small positive constant $\epsilon$.

In view of $d_{2}>D>s / \mu_{1}$, we can find a sufficiently small $\epsilon_{0}>0$ such that $d_{2} \mu_{1} \geq s+\epsilon_{0} M$. Let $d^{*}=\left(1 / \mu_{1}\right)(r+M / \epsilon)$, then

$$
\nabla(N-\bar{N})=\nabla(P-\bar{P})=0
$$

and $(N, P)$ must be a constant solution. This completes the proof.

3.3. Existence of the Nonconstant Positive Solutions. In this subsection, we shall discuss the existence of the positive nonconstant solution of model (9).

Unless otherwise specified, in this subsection, we always require that $m<r(a+h)$ holds, which guarantees that model (9) has the unique positive constant solution $E^{*}=\left(N^{*}, P^{*}\right)$. From now on, we denote $\mathbf{w}=(N, P)^{T}$ and $\mathbf{w}_{0}=E^{*}$.

Let $\mathbf{X}$ be the space defined in (25) and let

$$
\mathbf{X}^{+}=\{(N, P) \in \mathbf{X} \mid N, P>0 \text { on } C(\bar{\Omega})\} .
$$

We write model (9) in the following form:

$$
\begin{gathered}
-\Delta \mathbf{w}=\mathscr{G}(\mathbf{w}), \quad \mathbf{w} \in \mathbf{X}^{+}, \\
\partial_{\nu} \mathbf{w}=0 \quad \text { on } \partial \Omega,
\end{gathered}
$$

where

$$
\mathscr{G}(\mathbf{w})=\left(\begin{array}{c}
\frac{N}{d_{1}}\left(\frac{r(K-N)}{K+c N}-\frac{m P}{N+a P}\right) \\
\frac{s P}{d_{2}}\left(1-\frac{h P}{N}\right)
\end{array}\right) .
$$


Then $\mathbf{w}$ is a positive solution of model (65) if and only if w satisfies

$$
\mathscr{F}(\mathbf{w})=\mathbf{w}-(\mathbf{I}-\Delta)^{-1}\{\mathscr{G}(\mathbf{w}+\mathbf{w})\}=0, \quad \text { in } \mathbf{X}^{+},
$$

where $(\mathbf{I}-\Delta)^{-1}$ is the inverse operator of $\mathbf{I}-\Delta$ subject to the zero-flux boundary condition. Then

$$
\nabla \mathscr{F}\left(\mathbf{w}_{0}\right)=\mathbf{I}-(\mathbf{I}-\Delta)^{-1}(\mathbf{I}+\mathscr{A}),
$$

where

$$
\mathscr{A} \triangleq \nabla \mathscr{G}\left(\mathbf{w}_{0}\right)=\left(\begin{array}{cc}
\frac{J_{1}}{d_{1}} & -\frac{m h^{2}}{d_{1}(a+h)^{2}} \\
-\frac{s}{d_{2} h} & -\frac{s}{d_{2}}
\end{array}\right) .
$$

If $\nabla \mathscr{F}\left(\mathbf{w}_{0}\right)$ is invertible, by Theorem 2.8.1 of [28], the index of $\mathscr{F}$ at $\mathbf{w}_{0}$ is given by

$$
\operatorname{index}\left(\mathscr{F}, \mathbf{w}_{0}\right)=(-1)^{\gamma},
$$

where $\gamma$ is the multiplicity of negative eigenvalues of $\nabla \mathscr{F}\left(\mathbf{w}_{0}\right)$.

On the other hand, using the decomposition (26), we have that $\mathbf{X}_{i}$ is an invariant space under $\nabla \mathscr{F}\left(\mathbf{w}_{0}\right)$ and $\xi \in \mathbb{R}$ is an eigenvalue of $\nabla \mathscr{F}\left(\mathbf{w}_{0}\right)$ in $\mathbf{X}_{i}$, if and only if, $\xi$ is an eigenvalue of $\left(\mu_{i}+1\right)^{-1}\left(\mu_{i} \mathbf{I}-\mathscr{A}\right)$. Therefore, $\nabla \mathscr{F}\left(\mathbf{w}_{0}\right)$ is invertible, if and only if, for any $i \geq 0$ the matrix $\mu_{i} \mathbf{I}-\mathscr{A}$ is invertible.

Let $m\left(\mu_{i}\right)$ be the multiplicity of $\mu_{i}$. For the sake of convenience, we denote

$$
H(\mu)=\operatorname{det}(\mu \mathbf{I}-\mathscr{A}) .
$$

Then, if $\mu_{i} \mathbf{I}-\mathscr{A}$ is invertible for any $i \geq 0$, with the same arguments as in [29], we can assert the following conclusion.

Lemma 11. Assume that, for all $i \geq 0$, the matrix $\mu_{i} \mathbf{I}-\mathscr{A}$ is nonsingular, then

$$
\operatorname{index}\left(\mathscr{F}, \mathbf{w}_{0}\right)=(-1)^{\gamma}, \quad \text { where } \gamma=\sum_{i \geq 0, H\left(\mu_{i}\right)<0} m\left(\mu_{i}\right) \text {. }
$$

To compute index $\left(\mathscr{F}, \mathbf{w}_{0}\right)$, we have to consider the sign of $H(\mu)$. A straightforward computation yields

$$
H(\mu)=\mu^{2}-\theta_{1} \mu+\theta_{2}
$$

where $\theta_{1}=\left(d_{2} J_{1}-d_{1} s\right) / d_{1} d_{2}, \quad \theta_{2}=s(a r+h r-m)(a r+h r+$ $c m) / d_{1} d_{2} r(1+c)(a+h)^{2}$.

If $\theta_{1}^{2}-4 \theta_{2}>0$, then $H(\mu)=0$ has two positive solutions $\mu^{ \pm}$given by

$$
\mu^{ \pm}=\frac{1}{2}\left(\theta_{1} \pm \sqrt{\theta_{1}^{2}-4 \theta_{2}}\right)
$$

Theorem 12. Assume that $m(a r+c m+2 h r-a c r)>r^{2}(a+h)^{2}$ and $\theta_{1}^{2}-4 \theta_{2}>0$. If $\mu^{-} \in\left(\mu_{i}, \mu_{i+1}\right)$ and $\mu^{+} \in\left(\mu_{j}, \mu_{j+1}\right)$ for some $0 \leq i<j$, and $\sum_{k=i+1}^{j} m\left(\mu_{k}\right)$ is odd, then model (9) has at least one nonconstant solution.
Proof. By Theorem 10, we can fix $\bar{d}_{1}>d_{1}$ and $\bar{d}_{2}>d_{2}$ such that model (9) with diffusion coefficients $\bar{d}_{1}$ and $\bar{d}_{2}$ has no nonconstant solutions.

By virtue of Theorems 8 and 9, there exists a positive constant $\underline{C}, \bar{C}$ such that $\underline{C}<N, P<\bar{C}$.

Set

$$
\mathscr{M}=\{(N, P) \in C(\bar{\Omega}) \times C(\bar{\Omega}): \underline{C}<N, P<\bar{C} \text { in } \bar{\Omega}\},
$$

and define

$$
\Psi: \mathscr{M} \times[0,1] \longrightarrow C(\bar{\Omega}) \times C(\bar{\Omega})
$$

by

$$
\Psi(\mathbf{w}, t)=(\mathbf{I}-\Delta)^{-1}\{\mathbf{G}(\mathbf{w}, t)+\mathbf{w}\}
$$

where

$\mathbf{G}(\mathbf{w}, t)$

$$
=\left(\begin{array}{c}
\left(t d_{1}+(1-t) \bar{d}_{1}\right)^{-1}\left(\frac{r N(K-N)}{K+c N}-\frac{m N P}{N+a P}\right) \\
\left(t d_{2}+(1-t) \bar{d}_{2}\right)^{-1}\left(s P\left(1-\frac{h P}{N}\right)\right)
\end{array}\right) .
$$

It is clear that finding the positive solution of model (9) becomes equivalent to finding the positive solution of $\Psi(\mathbf{w}, 1)=0$ in $\mathscr{M}$. Further, by virtue of the definition of $\mathscr{M}$, we have that $\Psi(\mathbf{w}, t)=0$ has no positive solution in $\partial \mathscr{M}$ for all $0 \leq t \leq 1$.

Since $\Psi(\mathbf{w}, t)$ is compact, the Leray-Schauder topological degree $\operatorname{deg}(\mathbf{I}-\Psi(\mathbf{w}, t), \mathscr{M}, 0)$ is well defined. From the invariance of Leray-Schauder degree at the homotopy, we deduce

$$
\operatorname{deg}(\mathbf{I}-\Psi(\mathbf{w}, 1), \mathscr{M}, 0)=\operatorname{deg}(\mathbf{I}-\Psi(\mathbf{w}, 0), \mathscr{M}, 0) .
$$

Clearly, $\mathbf{I}-\Psi(\mathbf{w}, 1)=\mathscr{F}$. Thus, if model (9) has no other solutions except the constant one $\mathbf{w}_{0}$, then Lemma 11 shows that

$$
\begin{aligned}
\operatorname{deg}(\mathbf{I}-\Psi(\mathbf{w}, 1), \mathscr{M}, 0) & =\operatorname{index}\left(\mathscr{F}, \mathbf{w}_{0}\right) \\
& =(-1)^{\sum_{k=i+1}^{j} m\left(\mu_{k}\right)}=-1 .
\end{aligned}
$$

On the contrary, by the choice of $\bar{d}_{1}$ and $\bar{d}_{2}$, we have that $\mathbf{w}_{0}$ is the only solution of $\Psi(\mathbf{w}, 0)=0$. Furthermore, we have

$$
\operatorname{deg}(\mathbf{I}-\Psi(\mathbf{w}, 0), \mathscr{M}, 0)=\operatorname{index}\left(\mathbf{I}-\Psi(\mathbf{w}, 0), \mathbf{w}_{0}\right)=1 .
$$

From (79)-(81), we get a contradiction, and the proof is completed.

\section{Acknowledgments}

The authors would like to thank the anonymous referee for the very helpful suggestions and comments which led to improvements of our original paper. And this work is supported by the Cooperative Project of Yulin City (2011). 


\section{References}

[1] J. T. Tanner, "The stability and the intrinsic growth rates of prey and predator populations," Ecology, vol. 56, pp. 855-886, 1975.

[2] D. J. Wollkind, J. B. Collings, and J. A. Logan, "Metastability in a temperature-dependent model system for predator-prey mite outbreak interactions on fruit trees," Bulletin of Mathematical Biology, vol. 50, no. 4, pp. 379-409, 1988.

[3] C. S. Holling, "The functional response of predators to prey density and its role in mimicry and population regulation," Memoirs of the Entomological Society of Canada, vol. 45, supplement 45, pp. 5-60, 1965.

[4] M. P. Hassell, The Dynamics of Arthropod Predator-Prey Systems, Princeton University Press, Princeton, NJ, USA, 1978.

[5] P. H. Leslie and J. C. Gower, "The properties of a stochastic model for the predator-prey type of interaction between two species," Biometrika, vol. 47, pp. 219-234, 1960.

[6] R. M. May, Stability and Complexity in Model Ecosystems, Princeton University Press, Princeton, NJ, USA, 1973.

[7] S. B. Hsu and T. W. Huang, "Global stability for a class of predator-prey systems," SIAM Journal on Applied Mathematics, vol. 55, no. 3, pp. 763-783, 1995.

[8] A. Gasull, R. E. Kooij, and J. Torregrosa, "Limit cycles in the Holling-Tanner model," Publicacions Matemàtiques, vol. 41, no. 1, pp. 149-167, 1997.

[9] E. Sáez and E. González-Olivares, "Dynamics of a predator-prey model," SIAM Journal on Applied Mathematics, vol. 59, no. 5, pp. 1867-1878, 1999.

[10] R. Arditi, L. R. Ginzburg, and H. R. Akcakaya, "Variation in plankton densities among lakes: a case for ratio-dependent models," The American Naturalist, vol. 138, no. 5, pp. 287-296, 1991.

[11] R. Arditi and H. Saiah, "Empirical evidence of the role of heterogeneity in ratio-dependent consumption," Ecology, vol. 73, pp. 1544-1551, 1992.

[12] A. P. Gutierrez, "The physiological basis of ratio-dependent predator-prey theory: a metabolic pool model of Nicholson's blowflies as an example," Ecology, vol. 73, pp. 1552-1563, 1992.

[13] T. Saha and C. Chakrabarti, "Dynamical analysis of a delayed ratio-dependent Holling-Tanner predator-prey model," Journal of Mathematical Analysis and Applications, vol. 358, no. 2, pp. 389-402, 2009.

[14] R. Arditi and L. R. Ginzburg, "Coupling in predator-prey dynamics: Ratio-dependence," Journal of Theoretical Biology, vol. 139, no. 3, pp. 311-326, 1989.

[15] R. Arditi, N. Perrin, and H. Saiah, "Functional responses and heterogeneities: an experimental test with cladocerans," Oikos, vol. 60, no. 1, pp. 69-75, 1991.

[16] Z. Liang and H. Pan, "Qualitative analysis of a ratio-dependent Holling-Tanner model," Journal of Mathematical Analysis and Applications, vol. 334, no. 2, pp. 954-964, 2007.

[17] M. Banerjee and S. Banerjee, "Turing instabilities and spatiotemporal chaos in ratio-dependent Holling-Tanner model," Mathematical Biosciences, vol. 236, no. 1, pp. 64-76, 2012.

[18] F. E. Smith, "Population dynamics in Daphnia Magna and a new model for population growth," Ecology, vol. 44, pp. 651663, 1963.

[19] E. C. Pielou, An Introduction to Mathematical Ecology, Wiley, New York, NY, USA, 1969.

[20] T. G. Hallam and J. T. Deluna, "Effects of toxicants on populations: a qualitative approach III," Journal of Theoretical Biology, vol. 109, no. 3, pp. 411-429, 1984.
[21] K. Gopalsamy, M. R. S. Kulenović, and G. Ladas, "Environmental periodicity and time delays in a "food-limited" population model," Journal of Mathematical Analysis and Applications, vol. 147 , no. 2, pp. 545-555, 1990.

[22] W. Feng and X. Lu, "On diffusive population models with toxicants and time delays," Journal of Mathematical Analysis and Applications, vol. 233, no. 1, pp. 373-386, 1999.

[23] M. Fan and K. Wang, "Periodicity in a "food-limited" population model with toxicants and time delays," Acta Mathematicae Applicatae Sinica, vol. 18, no. 2, pp. 309-314, 2002.

[24] W. Chen and M. Wang, "Qualitative analysis of predator-prey models with Beddington-DeAngelis functional response and diffusion," Mathematical and Computer Modelling, vol. 42, no. 1-2, pp. 31-44, 2005.

[25] D. Henry, Geometric Theory of Semilinear Parabolic Equations, vol. 61 of Lecture Notes in Mathematics, Springer, Berlin, Germany, 1981.

[26] C.-S. Lin, W.-M. Ni, and I. Takagi, "Large amplitude stationary solutions to a chemotaxis system," Journal of Differential Equations, vol. 72, no. 1, pp. 1-27, 1988.

[27] Y. Lou and W.-M. Ni, "Diffusion, self-diffusion and crossdiffusion," Journal of Differential Equations, vol. 131, no. 1, pp. 79-131, 1996.

[28] L. Nirenberg, Topics in Nonlinear Functional Analysis, Courant Institute, 2nd edition, 2001.

[29] R. Peng, J. Shi, and M. Wang, "On stationary patterns of a reaction-diffusion model with autocatalysis and saturation law," Nonlinearity, vol. 21, no. 7, pp. 1471-1488, 2008. 


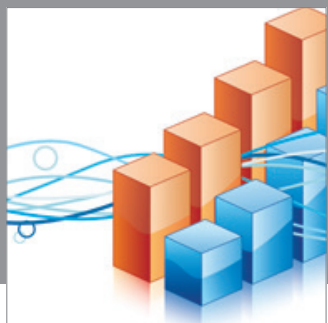

Advances in

Operations Research

mansans

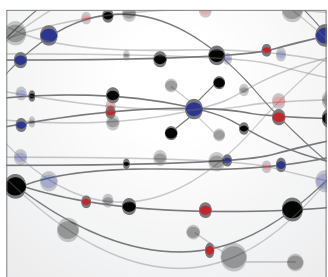

The Scientific World Journal
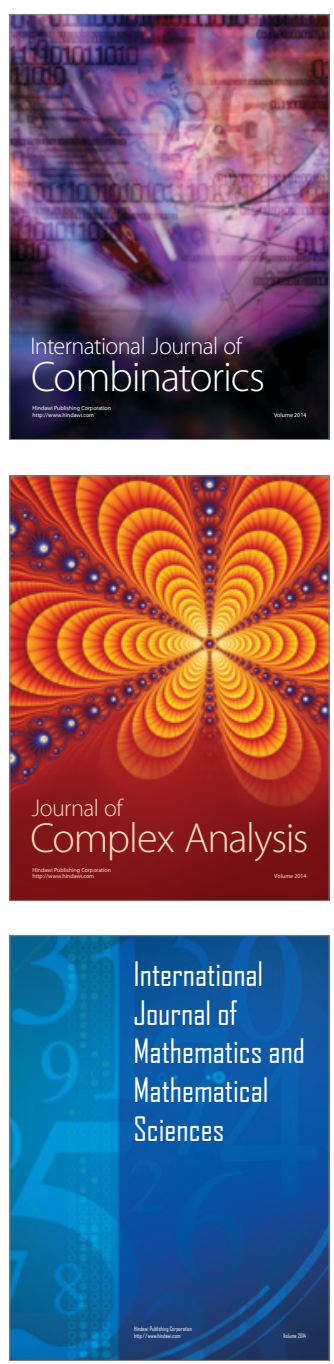
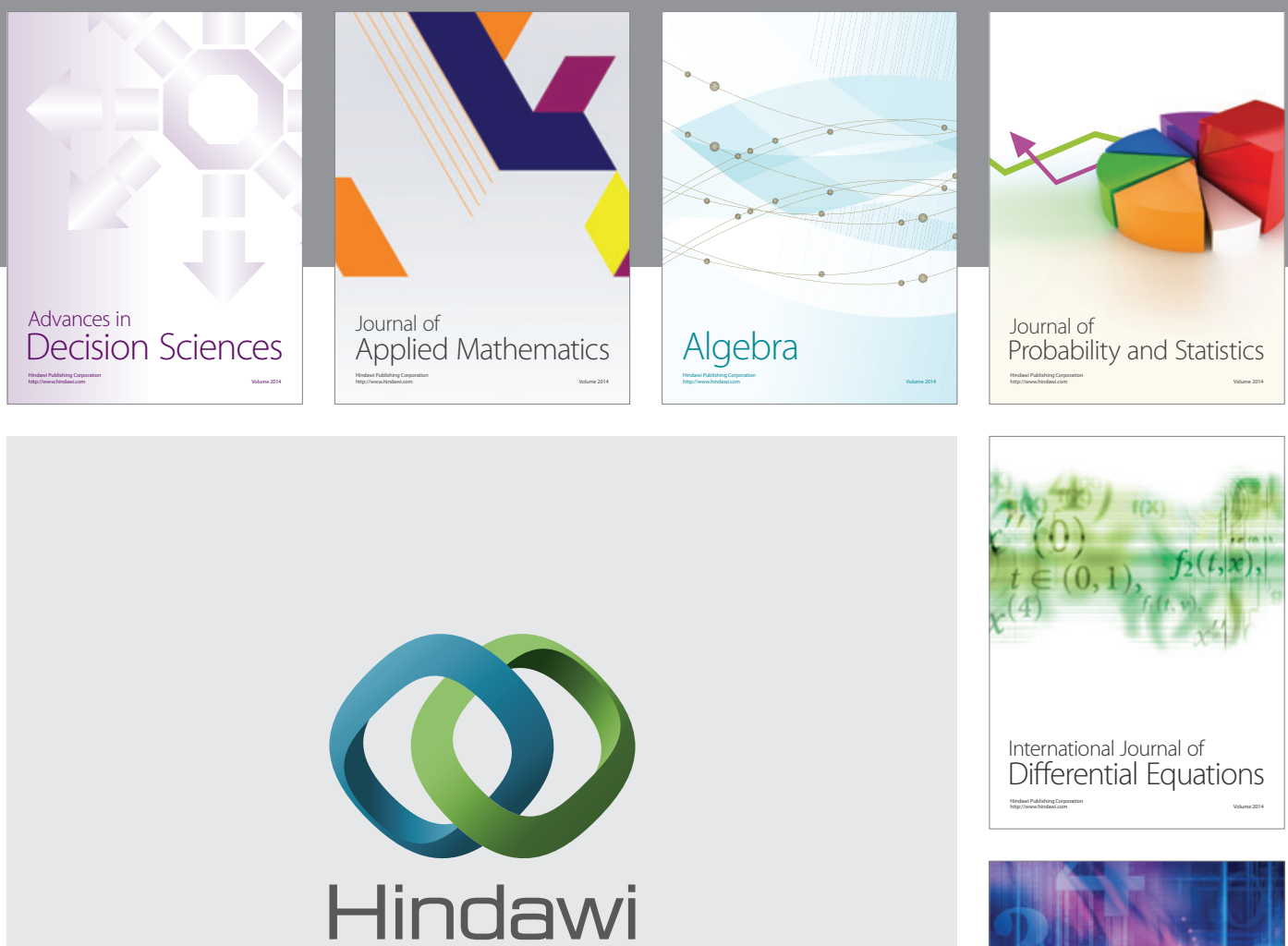

Submit your manuscripts at http://www.hindawi.com
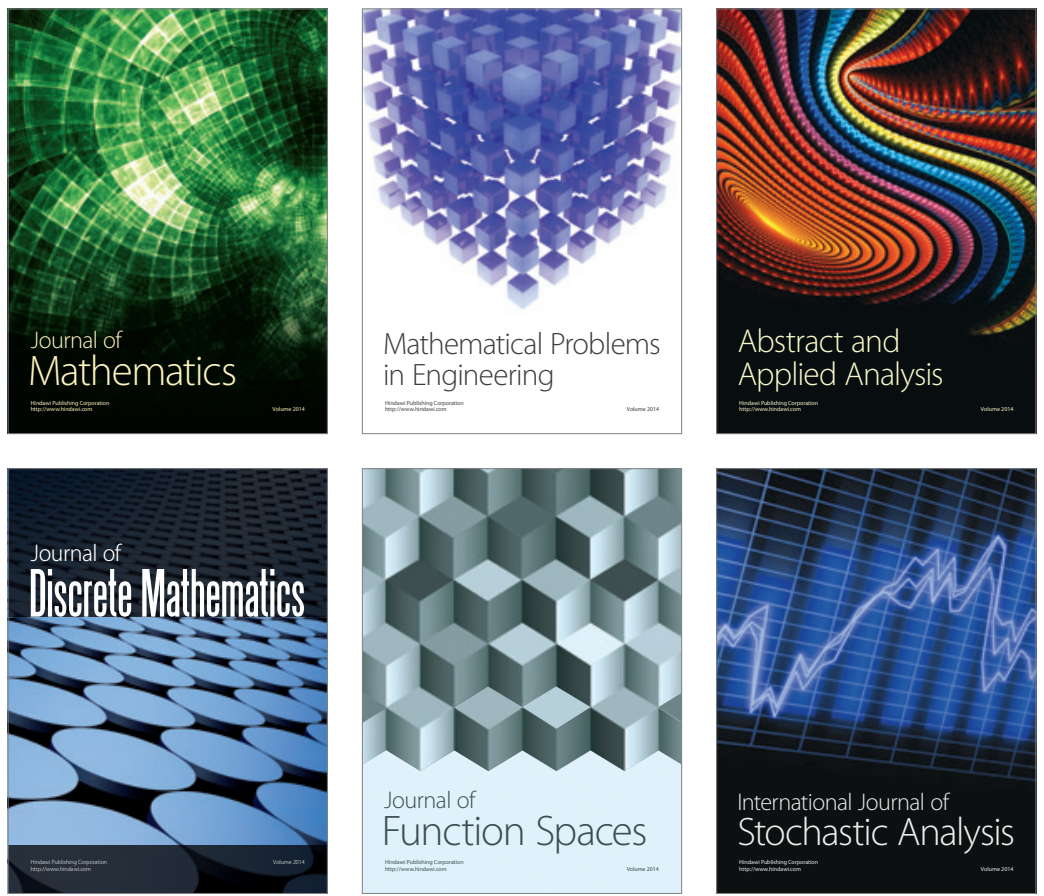

Journal of

Function Spaces

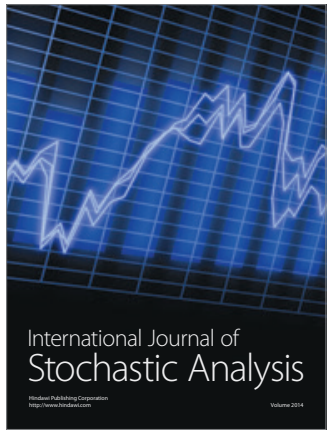

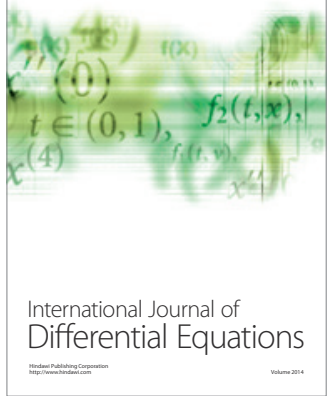
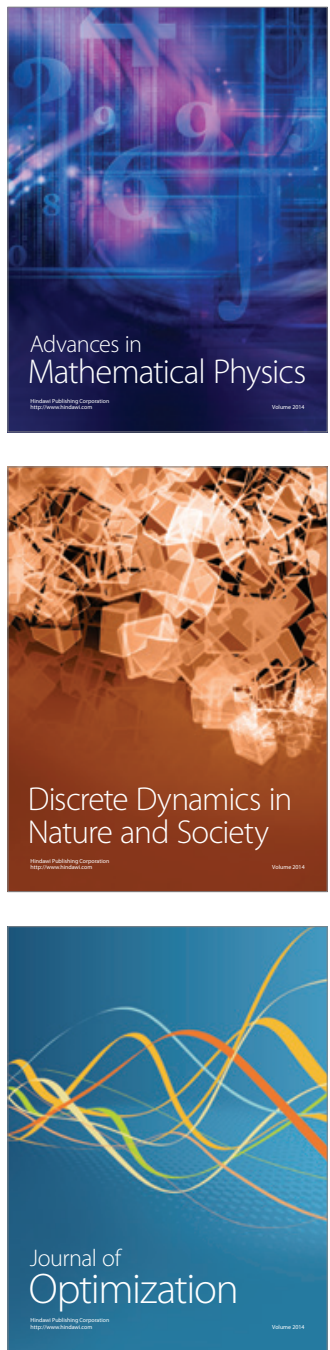